\title{
4.6 Siglenverzeichnis
}

Ausgaben

BHV Reigen. Zehn Dialoge. Geschrieben Winter 1896-97. 89.-99. Tausend. Berlin, Wien: Benjamin Harz=Verlag [1923/1924?].

EA Reigen. Zehn Dialoge. Geschrieben Winter 1896-97. Buchschmuck von Berthold Löffler. Wien, Leipzig: Wiener Verlag 1903.

JSV Reigen. Zehn Dialoge. Geschrieben Winter 1896-97. 44.-46. Tausend. Berlin: Verlag J. Singer \& CO. [1913?].

PD Reigen. Zehn Dialoge. Winter 1896/97. Als Manuscript gedruckt. Buchdruckerei Roitzsch vorm. Otto Noack \& Co. [1900].

SFV Reigen. Zehn Dialoge. 101.-104. Aufl. Berlin: S. Fischer Verlag 1931.

Edierte Texte

$\begin{array}{ll}\text { D } & \text { Drucktext (Grundlage: PD) } \\ \text { E } & \text { Entwurfskizze } \\ \text { H } & \text { Finale Textstufe; umfasst HF, HSz1-HSz10 } \\ \text { HF } & \text { Figurenliste } \\ \text { HSz1 } & \text { Szene 1 } \\ \text { HSz2 } & \text { Szene 2 } \\ \text { HSz3 } & \text { Szene 3 } \\ \text { HSz4 } & \text { Szene 4 } \\ \text { HSz5 } & \text { Szene 5 } \\ \text { HSz6 } & \text { Szene 6 } \\ \text { HSz7 } & \text { Szene 7 } \\ \text { HSz8 } & \text { Szene 8 } \\ \text { HSz9 } & \text { Szene 9 } \\ \text { HSz10 } & \text { Szene 10 } \\ \text { H'Sz9 } & \text { Textstufe zu Szene 9 } \\ \text { H'Sz10 } & \text { Textstufe zu Szene 10 } \\ \text { N } & \text { Notiz } \\ \text { TSz4 } & \text { Typoskript zu Szene 4 } \\ \text { U } & \text { Umschlag }\end{array}$


Unveröffentlichtes

NB

Notizbuch (CUL, A 193,2)

Zitierte Literatur

BI-HKA Arthur Schnitzler: Blumen. Historisch-kritische Ausgabe. Hrsg. v. Isabella Schwentner. Berlin, Boston: De Gruyter 2018 (Werke in historisch-kritischen Ausgaben).

BDB Börsenblatt für den deutschen Buchhandel.

Br I Arthur Schnitzler: Briefe 1875-1912. Hrsg. v. Therese Nickl u. Heinrich Schnitzler. Frankfurt a. M.: S. Fischer 1981.

Br II Arthur Schnitzler: Briefe 1913-1931. Hrsg. v. Peter Michael Braunwarth, Richard Miklin, Susanne Pertlik u. Heinrich Schnitzler. Frankfurt a. M.: S. Fischer 1984.

de Mendelssohn Peter de Mendelssohn: S. Fischer und sein Verlag. Frankfurt a. M.: S. Fischer 1970.

FBG-HKA Arthur Schnitzler: Frau Bertha Garlan. Historisch-kritische Ausgabe. Hrsg. v. Gerhard Hubmann u. Isabella Schwentner. Unter Mitarbeit v. Anna Lindner u. Martin Anton Müller. Berlin, Boston: De Gruyter 2015 (Werke in historisch-kritischen Ausgaben).

Fischer-Bw Samuel Fischer u. Hedwig Fischer: Briefwechsel mit Autoren. Hrsg. v. Dierk Rodewald u. Corinna Fiedler. Mit einer Einführung v. Bernhard Zeller. Frankfurt a. M.: S. Fischer 1989.

Hall Murray G. Hall: Der "Törleß"- und "Reigen"-Verleger. In: MusilForum 9 (1983), S. 129-149.

Heine $\quad$ Der Kampf um den Reigen. Vollständiger Bericht über die sechstägige Verhandlung gegen Direktion und Darsteller des Kleinen Schauspielhauses Berlin. Hrsg. und mit einer Einleitung v. Wolfgang Heine. Berlin: Rowohlt 1922.

Janz Rolf-Peter Janz: Zum Sozialcharakter des "süßen Mädels”. In: Ders. u. Klaus Laermann: Arthur Schnitzler: Zur Diagnose des Wiener Bürgertums im Fin de siècle. Stuttgart: Metzler 1977, S. 41-54; Ders.: "Reigen“. In: ebd., S. 55-75.

L-HKA Arthur Schnitzler: Liebelei. Historisch-kritische Ausgabe. Hrsg. v. Peter Michael Braunwarth, Gerhard Hubmann u. Isabella Schwentner. 2 Bde. Berlin, Boston: De Gruyter 2014 (Werke in historisch-kritischen Ausgaben).

LG-HKA Arthur Schnitzler: Lieutenant Gustl. Historisch-kritische Ausgabe. Hrsg. v. Konstanze Fliedl. Berlin, New York: De Gruyter 2011.

Pfoser I Alfred Pfoser, Kristina Pfoser-Schewig, Gerhard Renner: Schnitzlers ,Reigen'. Zehn Dialoge und ihre Skandalgeschichte. Analysen und Dokumente. Bd. 1: Der Skandal. Frankfurt a. M.: Fischer 1993. 
Pfoser II

Reinhardt-Bw

Rovagnati 2001

Rovagnati 2004

Schinnerer 1931

Schinnerer

1932/33

Schneider

St-HKA

Tb II

Tb III

Tb IV
Alfred Pfoser, Kristina Pfoser-Schewig, Gerhard Renner: Schnitzlers ,Reigen'. Zehn Dialoge und ihre Skandalgeschichte. Analysen und Dokumente. Bd. 2: Die Prozesse. Frankfurt a. M.: Fischer 1993

Der Briefwechsel Arthur Schnitzlers mit Max Reinhardt und dessen Mitarbeitern. Hrsg. v. Renate Wagner. Salzburg: Otto Müller 1971 (= Publikationen der Max-Reinhardt-Forschungsstätte II).

Gabriella Rovagnati: Arthur Schnitzler (1862-1931), Reigen'. In: Spiegel der Welt. Handschriften und Bücher aus drei Jahrtausenden. Eine Ausstellung der Fondation Martin Bodmer Cologny in Verbindung mit dem Schiller-Nationalmuseum Marbach und der Stiftung Museum Bärengasse Zürich. Hrsg. v. Martin Bircher in Zusammenarbeit mit Elisabeth Macheret-van Daele u. Hans-Albrecht Koch. Bd. 1. Cologny: Fondation Martin Bodmer u. Marbach: Deutsche Schillergesellschaft 2001 (= Marbacher Kataloge 55), S. 374-378.

Arthur Schnitzler: Ein Liebesreigen. Die Urfassung des "Reigen". Hrsg. v. Gabriella Rovagnati. Frankfurt a. M.: S. Fischer 2004.

Otto P. Schinnerer: The History of Schnitzler's Reigen. In: PMLA 46 (1931), 3, S. 839-859.

Otto P. Schinnerer: Systematisches Verzeichnis der Werke von Arthur Schnitzler. In: Jahrbuch deutscher Bibliophilen und Literaturfreunde 18/19 (1932/33), S. 94-121.

Gerd K. Schneider: Die Rezeption von Arthur Schnitzlers Reigen. 1897-1994. Text, Aufführungen, Verfilmungen, Pressespiegel und andere zeitgenössische Kommentare. Riverside: Ariadne 1995 (= Studies in Austrian Literature, Culture and Thought).

Arthur Schnitzler: Sterben. Historisch-kritische Ausgabe. Hrsg. v. Gerhard Hubmann. Berlin, Boston: De Gruyter 2012 (Werke in historisch-kritischen Ausgaben).

Arthur Schnitzler: Tagebuch 1893-1902. Unter Mitwirkung von Peter Michael Braunwarth, Konstanze Fliedl, Susanne Pertlik u. Reinhard Urbach hrsg. v. der Kommission für literarische Gebrauchsformen der Österreichischen Akademie der Wissenschaften, Obmann: Werner Welzig. Wien: Verlag der Österreichischen Akademie der Wissenschaften 1995.

Arthur Schnitzler: Tagebuch 1903-1908. Unter Mitwirkung von Peter Michael Braunwarth, Susanne Pertlik u. Reinhard Urbach hrsg. v. der Kommission für literarische Gebrauchsformen der Österreichischen Akademie der Wissenschaften, Obmann: Werner Welzig. Wien: Verlag der Österreichischen Akademie der Wissenschaften 1991.

Arthur Schnitzler: Tagebuch 1909-1912. Unter Mitwirkung von Peter Michael Braunwarth, Richard Miklin, Maria Neyses, Susanne Pertlik, Walter Ruprechter u. Reinhard Urbach hrsg. v. der Kommission für literarische Gebrauchsformen der Österreichischen 
Tb V

Tb VI

Tb VII

Tb IX

Tb $X$

TS-HKA

Urbach

Institutionen

CUL

DLA

NÖLA

TMW
Akademie der Wissenschaften, Obmann: Werner Welzig. Wien: Verlag der Österreichischen Akademie der Wissenschaften 1981. Arthur Schnitzler: Tagebuch 1913-1916. Unter Mitwirkung von Peter Michael Braunwarth, Richard Miklin, Susanne Pertlik, Walter Ruprechter u. Reinhard Urbach hrsg. v. der Kommission für literarische Gebrauchsformen der Österreichischen Akademie der Wissenschaften, Obmann: Werner Welzig. Wien: Verlag der Österreichischen Akademie der Wissenschaften 1983.

Arthur Schnitzler: Tagebuch 1917-1919. Unter Mitwirkung von Peter Michael Braunwarth, Richard Miklin, Susanne Pertlik u. Reinhard Urbach hrsg. v. der Kommission für literarische Gebrauchsformen der Österreichischen Akademie der Wissenschaften, Obmann: Werner Welzig. Wien: Verlag der Österreichischen Akademie der Wissenschaften 1985.

Arthur Schnitzler: Tagebuch 1920-1922. Unter Mitwirkung von Peter Michael Braunwarth, Susanne Pertlik u. Reinhard Urbach hrsg. v. der Kommission für literarische Gebrauchsformen der Österreichischen Akademie der Wissenschaften, Obmann: Werner Welzig. Wien: Verlag der Österreichischen Akademie der Wissenschaften 1993.

Arthur Schnitzler: Tagebuch 1927-1930. Unter Mitwirkung von Peter Michael Braunwarth, Susanne Pertlik u. Reinhard Urbach hrsg. v. der Kommission für literarische Gebrauchsformen der Österreichischen Akademie der Wissenschaften, Obmann: Werner Welzig. Wien: Verlag der Österreichischen Akademie der Wissenschaften 1997.

Arthur Schnitzler: Tagebuch 1931. Gesamtverzeichnisse 1879-1931. Unter Mitwirkung von Peter Michael Braunwarth, Susanne Pertlik u. Reinhard Urbach hrsg. v. der Kommission für literarische Gebrauchsformen der Österreichischen Akademie der Wissenschaften, Obmann: Werner Welzig. Wien: Verlag der Österreichischen Akademie der Wissenschaften 2000.

Arthur Schnitzler: Die Toten schweigen. Historisch-kritische Ausgabe. Hrsg. v. Martin Anton Müller. Unter Mitarbeit v. Ingo Börner, Anna Lindner u. Isabella Schwentner. Berlin, Boston: De Gruyter 2016 (Werke in historisch-kritischen Ausgaben).

Reinhard Urbach: Schnitzler-Kommentar zu den erzählenden Schriften und dramatischen Werken. München: Winkler 1974.

\section{Cambridge University Library}

Deutsches Literaturarchiv, Marbach a. N.

Niederösterreichisches Landesarchiv, St. Pölten

Theatermuseum, Wien 